\title{
Mathematical Models for Light Distribution in Rice Canopy
}

\author{
Liu Xin`an, Wang Bolun and Yu Guirui \\ (Shenyang Agricultural University, 120 Dongling, Shenyang, P. R. China)
}

\begin{abstract}
Albstract
In this paper, the regression effects, accuracies and figures of three mathematical models for simulating light vertical distribution in rice canopy were compared. The model $S z=S_{0} e^{a+b L A I(z)}$ was the best. The quantitative relationships between $a, b$ and $\operatorname{LAI}(z)$ accumulated to a height above the ground, solar altitudes were discussed. A universal available model for light distribution in crop canopy was proposed, and the error in utilizing the model was analysed. Meanwhile, daily changes in light intensity in representative layers of the canopy were described by harmonic analysis.
\end{abstract}

Key words: Rice, Canopy, Light distribution, Simulation, Model

Studying the relationship between light-distribution characteristics in crop canopy and canopy structure is of great practical significance for utilizing solar radiation energy effectively and increasing rice yields. This article aims at find a simple and useful mathematical model for light distribution within a crop canopy.

1. Materials and method of the experiment

August 15,1990 . It was a clear sky and the rice was in the primary stage of ripening. Light intensities within different layers were measured using a GE-1 illuminometer. (made at the Yinchuan Electrometer Factory, China) within a high - yield canopy (Shennong $91-418$, per unit area yield $10.35 \mathrm{t} / \mathrm{ha}$, crop height about $100 \mathrm{~cm}$, leaf area index $L A I=6.83$ ) and a general canopy (per unit area yield $9.21 \mathrm{t} / \mathrm{ha}$, crop height about $90 \mathrm{~cm}, \mathrm{LAI}=4.44$ ). The experiment began at sunrise and ended at sunset. (distance between layers is $10 \mathrm{~cm}$ )Particular time was taken around the exact hours. Meanwhile, the weight of green leaves, LAI and the specific leaf weights etc. were measured by the stratifying clip method so as to understand the vertical structure of the canopy.

The vertical distributions of light and daily changes within the rice canopy are analysed using a mathematical simulation method and was verified with experimental materials.

2. The vertical distribution characteristics of light within rice canopy.

1) The choice of mathematical model

The relationship between relative light intensities and the LAI $(z)$ of different layers was simulated in a computer by linear, quadratic and exponential function, mean values of $\mathrm{F}$ for 14 times obtained from the three models for high-yield canopy ( $5: 00-$ $18: 00)$ are $38.2,84.6$ respectively. The average values of the residue standard errors are $15.76,8.61,7.54$ respectively. Although the test results of $F$ with the three models all reach the significance level of 0.01 , the regression effects and fit accuracies of the exponential model are the best no matter for high - yield canopy or for general canopy. This can be seen from the compar-

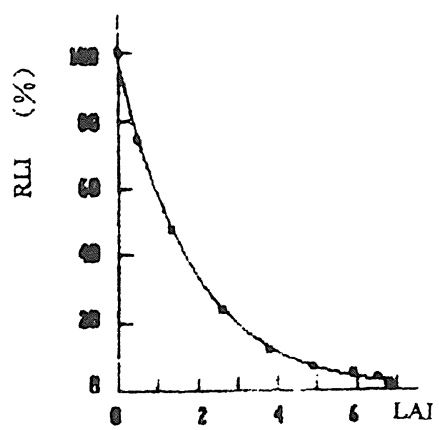

Fig. 1 The rebationship between the relative light intensity and LAI 
isons of the regression effects and accuracies of the three models. (for the exponential model: $F$ the highest and Se the lowest). The quadratic curve model is the second-best and the linear model the worst. Seen from a computer diagram, the exponential curve also best coincides with the dots from practical observation. (see Fig. 1)

The exponential model employed in this article is

$$
S_{z} / S_{0}=e^{a+b L A I(z)}
$$

Where: $S_{z}, S_{0}$ are the light intensities (klux) $Z \mathrm{~cm}$ and $0 \mathrm{~cm}$ from the surface of the canopy. For the purpose of easy calculation, the relative sunshine hours of different layers are expanded 100 times; for example, the practical value $7.5 \%$ for $7.5 . \mathrm{LAI}(\mathrm{z})$ is the leaf area index which is accumulated to $\mathrm{Zcm}$ below the upper canopy surface. $a$ and $b$ are parameters to be determined.

It is generally considered that the simple theoretical model $S_{z}=S_{0} e^{-b L A I(z)^{1)}}$ is suitable for the light distributions within crop canopy, where $b$ is the light-decreasing coefficient. Such a model assomes that the vertical distribution of light within the canopy is only influenced by the leaf area and that the distribution of leaf blades is homogeneous. But in fact, apart from the leaf area, many factors such as stem, colour of leaves, thickness of leaves and the incident angles of light all affect the reflection, absorption and transparent capacity of light. So there must be a parameter $a$ in the equation when the relationship between the relative light intensity and $\operatorname{LAI}(z)$ is simulated using exponential model ,i. e. :

$$
S z=S_{0} e^{a+b L A I(x)}
$$

From the time and space distributions of errors in the models, (tables omitted) below the active surface, (the height of $2 / 3$ crop height)errors of 63 percent of the simulated values are within the range of \pm $1 \%$, errors of 96 percent of the simulated values are within the range of $\pm 5 \%$, the average range of errors is $\pm 3 \%$. When it is before $8: 00$ or after $16: 00$, errors are relatively greater above the active surface. Thus this model is more applicable for layers under the active surface $(\operatorname{LAI}(z)>3)$, and also where the light intensity is comparatively weak, sometimes photosynthesis is restricted, therefore requiring further study it. It is thus seen that expression(1) is a simple and useful mathematical model for simulating the vertical distributions of light within rice canopy.

2) Discussions on parameters $a, b$ of the exponential model

Parameter $a, b$ are as shown in Table 1 .

Table 1 Parameter $a, b$ in exponential model

\begin{tabular}{c|cc|cc}
\hline Time & \multicolumn{2}{|c|}{ high-yield canopy } & \multicolumn{2}{|c}{ general canopy } \\
& $a$ & $b$ & $a$ & $b$ \\
\hline 5 & 4.5808 & -0.5958 & 4.3813 & -0.6763 \\
6 & 4.2715 & -0.4949 & 4.2403 & -0.7444 \\
7 & 4.1711 & -0.5160 & 4.3025 & -0.7681 \\
8 & 4.3279 & -0.5489 & 4.4619 & -0.7769 \\
9 & 4.5758 & -0.5269 & 4.6347 & -0.6879 \\
10 & 4.6585 & -0.4994 & 4.5861 & -0.5996 \\
11 & 4.6079 & -0.3326 & 4.5318 & -0.5227 \\
12 & 4.5670 & -0.3187 & 4.5746 & -0.4871 \\
13 & 4.6080 & -0.4165 & 4.3237 & -0.5352 \\
14 & 4.5585 & -0.4710 & 4.5233 & -0.6522 \\
15 & 4.6265 & -0.5392 & 4.3788 & -0.6931 \\
16 & 4.4926 & -0.5299 & 4.0376 & -0.6060 \\
17 & 4.1350 & -0.4684 & 3.9530 & -0.5270 \\
18 & 4.1832 & -0.2980 & 4.2282 & -0.3988 \\
\hline
\end{tabular}

Table 1 shows that the values of $a$ and $b$ vary with observational times. This in essence is the change of solar altitude. The incident angle of light follows it and finally results in the change of the vertical distribu- 
tions of light energy. The formula of solar altitude could be expressed as :

$$
\operatorname{Sin} h=\sin \varphi \sin \delta+\cos \varphi \cos \delta c a s t
$$

Parameter $a$ has a linear relationship with the sine of solar altitude, which is

$$
a=a_{1}+a_{2} \sinh
$$

$$
\text { When LAI }=6.83, a_{1}=4.0499, a_{2}=0.6739
$$

$$
\text { ( } n=13 R=0.89 * \text { *) }
$$

When LAI $=4.44, a_{1}=4.0591, a_{2}=0.5261$

$$
\left(n=12 R=0.64^{* *}\right)
$$

The average value is $a_{1}=4.0545, a_{2}=0.60$

$$
\text { ( } n=26 R=0.75^{*} \text { ) }
$$

Through tracing and analyzing the diagram, it can be found that the up-or-down trend of the curve of $b$ which varies daily runs opposite to that of $\sin 2 \mathrm{~h}$, their peaks and valley are going against each other. (diagram omitted)When a canopy is very thick, from $8: 00$ to $16: 00$, the correlation coeffeicient can reach the significant level of 0.05 . Then the expression is

$$
\begin{gathered}
b=b_{1}+b_{2} \sin 2 h \\
\text { Where } b_{1}=0.3810, b_{2}=0.9200 \\
\left(n=9, R=0.6734^{*}\right)
\end{gathered}
$$

When LAI is small or in the early morning, evening, solar altitude varies rapidly and negative correlation becomes weak with no significance.

3)The establishment of a universal available model

Solar altitude reflects the space distributional factors (geographical latitude $\varphi$ ) and time distributional factors, (annual variation $\delta$ and daily changes $t$ )so if a general applicable model is established which is available anywhere at anytime, the factor of solar altitudes must be introduced-i. e. : to establish a universal available model. The exact computational technique for solar altitude can be found in reference ${ }^{2)}$. Put (3), (4)into(1):

$$
S_{z}=S_{0} e^{a_{1}+a_{2} \sinh +b_{1} \operatorname{LAI}(Z)+b_{2} \sin 2 \operatorname{LLAI}(Z)}
$$

Where $a_{2}$ and $b_{1}$ are respectively the respons of the coefficients of $\sinh$ and LAI $(z) ; b_{2}$ could be considered as the interaction term of $\sinh$ and $\operatorname{LAI}(z) ; a_{1}$ is the affection of other factors. Their values are: $a_{1}=4$. $0545, a_{2}=0.6, b_{1}=0.381, b_{2}=-0.92$. Then, the expression (5) could be considered as the universal available model of light distribution within a crop canopy.

When calculating the relative light intensities among different layers within the LiZa rice canopy (leaf type and crop height similar to Shennong91) with expression(5), the standard error for calculating values of 40 experimental points is $4.39 \%$ t taking 5.5 percent of the experimental points, the error is within the range of $\pm 3 \%$; If takeing 75 percent of the points, the error is within $\pm 5 \%$. (table ommitted)It should be noted that there are 24 points below the level $2 / 3$ of the crop height, and the standard error is just \pm 2 . $20 \%$, moreover, it is just $\pm 1 \%$ for ten points. However when it is above the active surface level, the errors are relatively greater because the distribution of leaf blades is sparse and inhomogeneous.

\section{The daily changes of the light intensity within the rice canopy}

As to the daily changes, although the quadratic curve and perpendicular polynomial could be used for simulation, the application of harmonic analysis ${ }^{3}$ ) is clearer and more perceptible. Because the light intensity at horizontal surface is proportional to sinh, the daily changes of light intensity could be considered as a combination of some sine-waves and its expression is:

$$
Y i(t)=A_{0}+\Sigma A_{n} \sin \left(n \omega t i+\theta_{n}\right)
$$

Where (1) $\mathrm{A}_{0}$ is the mean value of these observational series and its unit is klux. Yi(t) is the estimated value for the same layer tested at different times and $i$ is the number of observation times. (2) $A_{n}$ is the half of the daily amplitude of light intensity wave for different periods. (3) $\omega$ is frequency (4)ti stands for the time of 
No. $i$ observation. (5) $\theta_{n}$ is the primary phase of the $n-t h$ wave. (6) represents fit degree.

Simulated formulae for different typical layers are shown as follows:

Top of the canopy:

$$
Y i(t)=60.14+63.74 \sin \left(\omega t i+272.3^{\circ}\right)
$$

Fit goodness of the basic wave (period length 16 hours)is $r_{1}=0.992$.

Active surface level

$$
Y i(t)=12.25+17.69 \sin \left(\omega t i+277.1^{\circ}\right)+80.9 \sin \left(2 \omega t i+283.1^{\circ}\right)
$$

Period length is also 16 hours, fit degree $r_{1}=0.767$, If 8 hours are added to the 16 hours period length, the fit degree can reach $\mathrm{r}_{2}=0.927$

Bottom of the canopy ( $80 \mathrm{~cm}$ below the canopy surface):

$$
\begin{aligned}
Y i(t)= & 2.9+4.17 \sin \left(\omega t i+274.5^{\circ}\right)+2.34 \sin \left(2 \omega t i+289.8^{\circ}\right) \\
& +12.7 \sin \left(4 \omega t i+314.0^{\circ}\right)
\end{aligned}
$$

Fit degree of one periodical wave is only 0.583 for two periodical waves $r_{2}=0.766$, for three waves the integrated $r_{3}=0.82$

Expression (7), (8) and (9) show: Average daily light intensity decreases down the canopy surface $(60.14 \rightarrow 12.25 \rightarrow 2.9 \mathrm{klux})$; Daily amplitude of the basic wave $\left(2 \times \mathrm{A}_{1}\right)$ becomes smaller $(2 \times 63.72 \rightarrow 2$ $\times 17.69 \rightarrow 2 \times 4.17)$ and the fit degree also decreases gradually $(0.992 \rightarrow 0.767 \rightarrow 0.583)$. Between the top level and the active surface, the primary phase of the basic wave becomes stagnant. The situation under the active surface is complicated and not in accordance with the rules described above. This can be explained like this: The top surface of the canopy and upper layers are more affected by the daily solar radiation variation rule of the sun than the lower layers which is affected to a

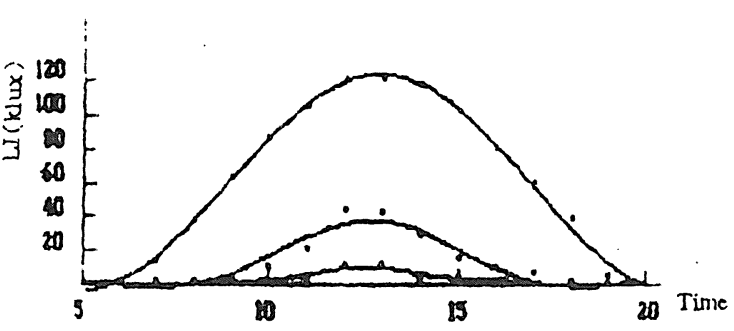

Fig. 2 The daily changes of the light intensity within different typical layers great extent by the vertical structure of the canopy. Comparing the errors between the simulated curves and the practical observation points. (see Fig. 2)

\section{Conclusion}

1) When simulations of light distributional characteristics within a crop canopy are studied, it is more suitable to choose a period in which the canopy grows at its fastest speed, (LAI greatest)i. e. , from $8: 00$ to $16: 00$ during the day.

2) $S_{z} / S_{0}=e^{a+b L A I(z)}$ is the best form for the simulations of the vertical distribution of light energy within a canopy since it has the best simulation effect and a higher accuracy.

3) Further study of the relationship between parameters $a, b$ and solar altitude, and the internal structure of a canopy makes it possible to establish a universal available model for the light distribution within crop fields which woold be applicable anywhere at anytime. This article gives a trial and the error of the simulation for $75 \%$ points within a range of $\pm 5 \%$.

4)It is a simple method and has an obvious physical significance to study the daily change characteristics of light intensity or radiation intensity within the typical layers in a crop canopy using the harmonic analysis.

The simulation of light distribution within a crop canopy is a complicated problem, the above conclusion still needs further studying and modification so as to make it perfect.

\section{References}

1)Weng Doming etal. 1981: 《Microclimate and the Crop Field Microclimate 》. 220-270.

2)Ichiro Watanabe, 1987 : 《Experiment Method of Agricultural Environment 》. 265-270.

3)Beijing Agricultural University, 1980: Textbooks of 《Agricultural Meteorological》. 32-41 